\title{
Experimental investigation of high speed SLD impact
}

\author{
Virginel Bodoc, Pierre Berthoumieu
}

\author{
ONERA / DMPE, Université de Toulouse, F-31055 Toulouse - France
}

\begin{abstract}
This paper proposes an experimental investigation of fast impinging large droplets in non-icing conditions. Two main aspects of the impact event are analyzed and discussed: the impact dynamics as a function of the surface nature and the deposition rate of the liquid on the impingement surface for various conditions. The data has been recorded and characterized at ambient pressure and a temperature of the air between 5 and $10^{\circ} \mathrm{C}$ using a vertical wind/droplet tunnel. To avoid the droplets evaporation the relative humidity was controlled. The morphology of impact was studied by backlighted imagery and quantitative results were obtained by image analysis. The deposition rate was obtained weighting the water accumulated on the impingement plate. Examination of splashing events images obtained on a clean surface and on blotter paper shows important differences in terms of secondary drop generation. The measurement of the deposition rate confirmed that the dynamics of drop impingement on blotter paper is not the same as impingement on a clean surface. These differences may significantly impact the development of models for icing applications.
\end{abstract}

\section{Introduction}

One of the main concerns of Supercooled Large Drops (SLD) icing is that existing ice protection systems cannot adequately remove all of the accreted ice since larger drops, with a large inertia, which can impinge beyond the physical limits of protection systems. The other concern is that, after impact, the ejected/rebounded drops can potentially accrete in areas of the aircraft that have no ice protection.

Although drop impact on walls has already been the subject of numerous studies in the past, which have enabled a certain level of understanding, the physics of the impact requires further investigation to complete this knowledge. A deep understanding and an accurate modelling of drop impact is a prerequisite for simulating icing phenomena related to the SLD.

In this work, the liquid structure dynamics during the impact of water drops on a rigid dry surface at high velocities is investigated experimentally. The kinematic parameters (size, velocity, impact angle) of the drops and the impingement surface are varied in order to account for parameters relevant for flight conditions.
The current methods used to estimate water collection on aircraft surfaces are based on water droplet trajectory calculation and an empirical model to account for the droplet/surface impact. In this paper, we propose a direct approach consisting in the experimental investigation of large drops impacting on a planar surface for temperatures of the air between 5 and $10^{\circ} \mathrm{C}$. Both qualitative and quantitative analyses are performed for drops during the impact. For that purpose, two experimental approaches were applied: rapid visualization and deposition rate evaluation by recovery/weighing.

The description of the splashing mechanism addresses a fundamental level, however the conclusions may extend to the understanding of water dynamics on airfoil geometries. The results of this work should be accepted as a first step before proceeding to perform tests in a freezing tunnel to improve the understanding of the SLD behavior under icing conditions.

The objective of this paper is to document large droplet impingement dynamics for non-icing conditions using different investigation methods. A review of the representative publications on the dynamics of SLD impact is reported in the next section. The third section presents the experimental setup and the investigation techniques. The fourth section presents qualitative and quantitative results on splash dynamics and a comparison to the available experimental data. The paper ends with concluding remarks.

\section{Literature review}

Usually, ice accretions result from small water drops (less than 50 microns in diameter) that freeze upon impact with aircraft surfaces. The resulting ice shapes can be estimated by ice accretion codes as LEWICE [1], TRAJ2D/IGLOO [2], CANICE [3] that are based on the droplet tracking calculation.

Ice accretions due to SLD (more than 50 microns in diameter), however, can be subject to additional effects resulting in ice shapes that could be quite different from the simulated results. Actually, in flight conditions, a boundary layer develops on either side of the geometry. Hence, except for the stagnation point area, the drops encounter a boundary layer structure with a strong shear component. Large drops are more susceptible to distortion and break-up before impact. Upon impact it may deposit on the surface as ice, or splash, creating secondary drops, or entirely rebound into the airflow. The secondary drops or the rebound drops may re-impinge on the surface of the airfoil if the airflow fails to carry them away [4]. 
From the point of view of SLD impingement on an aerodynamic surface, the experimental investigation focused mainly on the final water retention [5] [6], with few insights into the drop behavior before, during and after impact. Impingement tests with large drops at ambient temperature have demonstrated that droplet splashing and droplet breakup before impingement can have a significant impact on the impingement characteristics of the aerodynamic surfaces. Moreover, as was shown by various works [2], [8] the deposition models fail to correctly describe water accumulation in regions close to the impingement limits.

In developing simulation tools for icing applications, it is important to capture the effect of large drop impingement dynamics on the ice accretion process. Some of the models developed for SLD impact ([1], [2] [3], [7]) were calibrated and validated using the experimental data of Papadakis et al. [6]. The experimental data (impingement efficiency) was obtained using a dye-tracer technique, which involved spraying a known concentration of dye-drops towards the target airfoil, which was covered with blotter paper. The amount of dye deposited on the paper was directly related to the water impingement distribution. The limitation of this method comes from its representativeness of the real application. Hence, the authors did not establish whether the dynamics of drop impingement on blotter paper is the same as impingement on a clean airfoil surface or on an ice layer.

A list of the various models for SLD impact on a rigid surface was made by Trontin and Villedieu [2]. Moreover, they proposed a methodology to derive a new empirical mass loss model that can be used both for small and large drops (within the range of SLD). This allows the influence of the normal kinetic energy, which has a dominating effect close to the leading edge, to be distinguished from the influence of the angle of incidence, which is the most influent parameter close to the impingement limits. The experimental data used for calibration and validation were taken from the tests performed in the NASA Icing Research Tunnel [5], [6]. The quantity measured in the experiment is the collection efficiency and it is measured using the blotter paper strips installed on the airfoils. Due to the fact that the impingement efficiency cannot be directly measured, it is not possible to extract the values of the deposition rate from the experimental data only. A numerical approach must be used in addition to determine the impingement efficiency. Trontin and Villedieu [2] used a droplet trajectory solver to numerically compute this quantity from the operating conditions for different types of airfoils. Hence, by combining the experimental data base with a numerical approach it was possible to simultaneously know the size and velocity of the drops, the impinging mass flow rate, and the deposition mass flow rate. From the last two quantities the deposition rate was computed at each point of the tested profile. The assessment of this model for different operating conditions and airfoil profiles demonstrated a significant improvement of the deposition rate compared to the full deposition rate. Nevertheless, they pointed out that a comprehensive model accounting for the large drops should take into account the influence of the aerodynamic flow in the vicinity of the impinging point.

The impingement dynamics of drops on a dry or wet rigid surface plays an important role in the ice accumulation on aircraft. Many efforts have been made to understand the mechanisms of droplet impingement on a solid surface and to develop empirical models. For an overview of the collision dynamics of high-speed drops, the paper by Yarin [9] can be referred to. This review was later completed by Josserand and Thoroddsen [10]. The research work relevant to the

Page 2 of 11 physics of supercooled large drops and their icing behavior on aircraft was also reviewed by Li et al. [4].

The dynamics of the impact exhibit different behaviors depending on the impact and substrate parameters, ranging from spreading to splashing, jetting and bouncing [10], [11].

Various studies attempted to investigate the droplet splashing mechanism in low-speed regions. The droplet impact on solid surface was experimentally studied by Mundo et al. [12] with the objective of formulating an empirical model describing the deposition and the splashing process. The test matrix involved different droplet diameters, velocities, impingement angles, viscosities and surface tensions.

Following Guo et al. [13] the splashing occurs through a two-stage process. In the first stage the liquid droplet ejects a thin sheet before it contacts the surface; in the second stage the sheet contacts the surface and viscous forces can deflect the sheet upwards to cause a splash. It was numerically confirmed that a thin sheet lifts off to cause splashing.

Following Cossali et al. [14], for the temporal phase the splashing can be subdivided into: a) drop impact with lamella formation and a possible prompt splash, b) corona formation, c) jet formation and breakup generating secondary drops, d) corona collapse and/or possible non-elastic rebound. Usually, for drops impacting on a rigid and dry surface at ambient pressure, several aspects are discussed, such as the drop spread, the spreading/splashing threshold and the dynamics of splashing. The spreading/splashing threshold was characterized by the dimensionless group $K=W e \cdot O h^{-0.4}$ [12] [14], which incorporated the drop size and velocity, viscous stress and surface tension. When $K$ is greater than a value determined from the surface roughness, the splashing appears. The critical velocity of the drop required to produce a splash increases as the surface roughness decreases. For a clean surface, the splash is expected for $K>3500$.

From the works of Rioboo et al. [15], the nature of the splash has been divided into two categories, prompt and corona splash. A prompt splash releases secondary drops directly from the breakup of the tip of the advancing lamella, whereas in the corona splash the intact lamella rises away from the surface forming a corona like structure, which subsequently breaks up into small drops. The corona splash was observed on smooth surfaces, while the prompt splash was produced on very rough surfaces. Roughness inhibits thin-sheet formation even though it also increases prompt splashing at the advancing contact line.

Surrounding air was not initially considered to affect the droplet impact. However, Xu et al. [16] and Latka et al. [17] discovered experimentally that decreasing the ambient air pressure (and, as a consequence, decreasing the air density) completely suppresses the splashing. This was confirmed numerically by Guo at al. [13].

After the analysis of the main publications on the topic of large droplet impact, it seems that most of the experimental and numerical studies deal with impact speeds lowers than $50 \mathrm{~m} / \mathrm{s}$ and large droplet diameters, in the millimeter range. In most of the experimental studies, drops fall under gravity, with the height of the setup being varied in order to adjust the impingement velocity. This configuration ensures reproducibility of the shapes and dynamical stability only for drops traveling at low velocities. Given that fast-traveling drops may be exposed to shear and may deform, most of the investigations concerning velocities above $10 \mathrm{~m} / \mathrm{s}$ are restricted to drops smaller 
than $100 \mu \mathrm{m}$. In this work, however, we aim to study the impingement regimes at higher velocities and greater diameters of the drops, which are more representative of real conditions.

\section{Experimental setup}

To achieve the test conditions of high-speed impact, a vertical air/droplet tunnel was developed at ONERA. Previous tests [18] carried out in a wind tunnel have demonstrated the need to use a vertical installation to overcome the gravity effect, which modifies the trajectory of the drops as a function of their mass and speed of the carrier flow.

A detailed description of the setup was already provided in [19]. Only basic elements will be introduced here for completeness. Its main feature consists in the acceleration of liquid drops, in order to reproduce the realistic conditions of drop/wall collision. The chosen solution is to inject calibrated drops into an accelerating air stream. The main challenge of this approach is to accelerate the drops without breaking them. There are numerous studies in the literature on the secondary fragmentation of drops by aerodynamic effects. The aerodynamic Weber number is generally used to define the limit below which a drop maintains its cohesion. This value is fixed between 10 and 12 according to the authors; beyond this value the breakup of the drop occurs.

A general view of the test bench is shown in Figure 1. The inlet plenum serves both to condition air supplied by the local 80-bar network into the tunnel and to provide a suitable space for the monodispersed droplet generator and its displacement mechanism. The flow rate is measured upstream by a Coriolis flowmeter and controlled by a control valve. In order to reduce the air inlet speed, the airflow is injected through the four faces of the plenum. A grid creating a slight pressure drop and a honeycomb are placed in the plenum to break the large turbulent structures and homogenize the air flow before entering into the channel. The liquid injector tip is also placed at the entrance of the channel and a stream of monodisperse water drops are released at the center of the tunnel section. The droplet release location was adjusted until the drops impinged on the target installed at the exit of the tunnel.

The channel consists in a double convergent of square cross section, linearly arranged. The first convergent rapidly accelerates the air in the vicinity of the injector, in order to entrain the drops as rapidly as possible away from the injector nozzle and to avoid coalescence. The second convergent accelerates the air more gradually and reduces the slip speed between the drops and the carrier flow. The global length of the channel was defined by the available height of the laboratory room.

At the bottom of the channel there is the $50 \mathrm{~mm}$ square end of the contraction. The test area is placed after the exit of the channel. The flow achieved was adequate to observe drops in the SLD range in diameter at velocities up to $90 \mathrm{~m} / \mathrm{s}$.

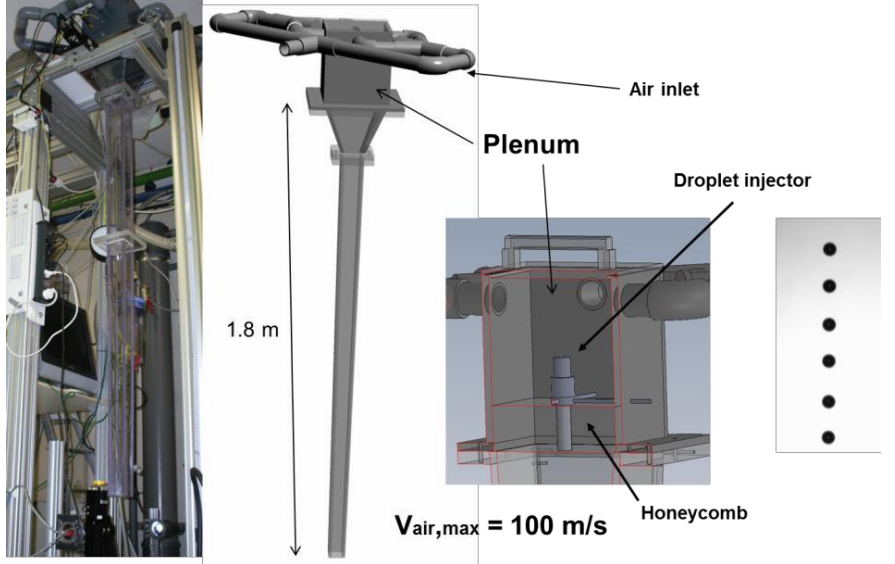

Figure 1. Experimental facility

A numerical simulation tool was used to estimate the kinematic characteristics of the drops in the carrier flow. The simulation is based on a balance of aerodynamic forces on drop and gravity to obtain the drop velocity at each location in the channel. As an example, for a drop of $200 \mu \mathrm{m}$ and for an air velocity at the output of $120 \mathrm{~m} / \mathrm{s}$, the drop reaches a final velocity of $85 \mathrm{~m} / \mathrm{s}$ without breakup $(W e<5)$. However, for this, the speed equilibrium between the air and the drop cannot be attained for the given channel length.

The monodisperse droplet generator used for this work delivers drops with identical size and spacing in a large range of diameters. Drops are generated by applying a longitudinal vibration to a thin cylindrical jet of liquid. The excitation is provided by a piezoelectric ceramic driven by a function generator. The jet expands and contracts at the excitation frequency forming individual drops, a phenomenon also known as Rayleigh jet breakup.

The droplet size is directly linked to the excitation frequency imposed at the ceramic, the diameter of the liquid jet which is fixed by the exit pinhole of the injector and the liquid flow rate.

The excitation frequency should be adapted to the liquid flow rate and the injector diaphragm to obtain a monosized drop stream. The diameter of the drops depends essentially on the diameter of the pinhole and can be slightly modified by varying the excitation frequency. The initial velocity of the drop depends essentially on the flow rate, and therefore on the injection pressure. The use of this injector makes it possible to generate drops with a speed varying between 5 and $10 \mathrm{~m} / \mathrm{s}$, by modulating the injection pressure of the water. It is worth noticing that, although the droplet injector produced a monodispersed stream of drops, a polydisperse cloud of drops was observed arriving in the working section. Turbulent dispersion and coalescence in the channel could be at the origin of this result.

\section{Instrumentation for splash visualization}

Several means have been implemented for drop impact characterization. In a first approach, a qualitative analysis was performed by visualization. This analysis was completed by a quantitative characterization that concerns the estimation of the deposition rate on the surface.

For the study of the impact a modified aluminum cylinder was used. As can be seen in Figure 2, it was slightly machined in order to produce a flat impingement surface with two different surface 
roughness. An articulation allows the impingement angle to be varied. The target was located at about $100 \mathrm{~mm}$ from the channel outlet.

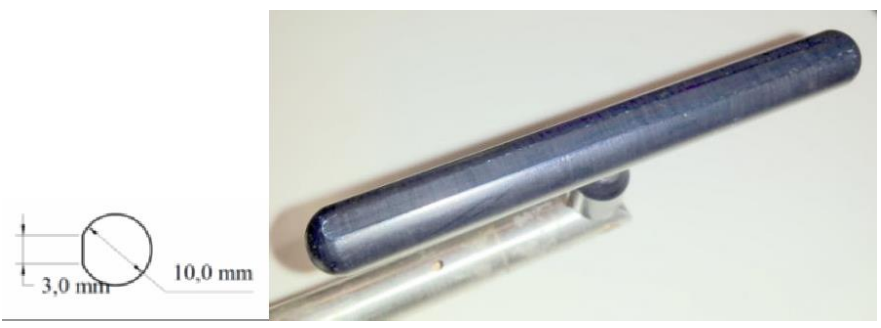

Figure 2. Test target for visualization

Phenomena observation is an essential step in their characterization and to understand how they unfold. All visualizations of the drop impact are performed by backlit imagery, the camera and the light source being placed on either side of the drop. Under these conditions, the drops appear dark on a light background. Due to the fact that the drops of water are traveling with a high velocity, it is necessary to freeze their movement to avoid a blur effect. This is achieved by using very short light pulses combined with a fast shutter gate on the camera.

For the observation of the impact dynamics, a JAI AD $131 \mathrm{GE}$ camera was used (Figure 3). Its main feature is that it can record three successive images with time intervals that may be as short as $1.5 \mu \mathrm{s}$. The overall recording rate of the image triplets is $30 \mathrm{~Hz}$. This camera offers a resolution of 1.296 x 966 pixels with $3.75-\mu \mathrm{m}$ sides. To achieve a magnification high enough to observe water drops before and during impact, the camera was equipped with a $180 \mathrm{~mm}$ SIGMA macro lens. The resulting field of view was $6.5 \times 4.5 \mathrm{~mm}$ with a pixel size of $5 \mu \mathrm{m}$.

For lighting with very short flashes, a CAVILUX laser illumination system was used. It provides flashes with durations as short as $20 \mathrm{~ns}$. The light beam was focused to send a maximum of energy into the camera.

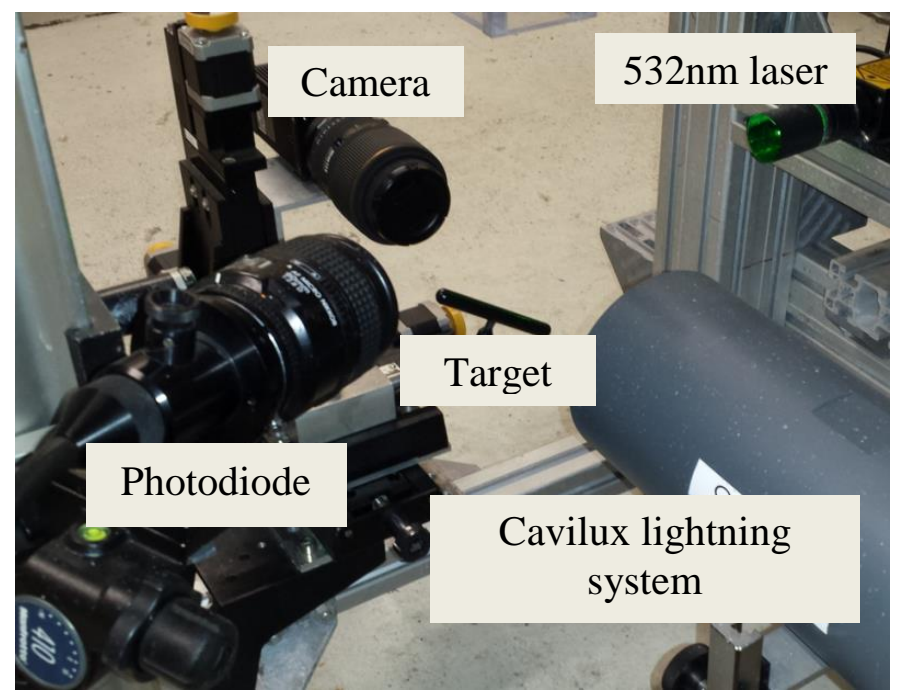

Figure 3. Optical setup for splash visualization
To avoid the effect of multiple droplets impinging and the high density of drops produced by splashing, a camera trigger needs to be used in addition to a small field of view and a small depth-of-field. Moreover, in the free recording mode of the camera it would be impossible to associate to each image the time elapsed from the impingement moment. For these reasons, it is compulsory to use an external trigger for the camera/lightning source assembly. The trigger scheme is shown in Figure 4. For the needs of this work, it was chosen to use the drop detection as the trigger source. For this purpose, a laser beam placed few centimeters before the impingement location lights the drops. The result is the light scattering by Mie process at the wavelength of the laser beam. A photosensitive element appropriately placed collects the light scattered by the well-centered drops. This signal is filtered and conditioned in order to use it as trigger source. A delay generator was placed between the photosensitive element and the camera, in order to finely account for the time elapsed between the drops crossing the laser beam and reaching the impingement location.

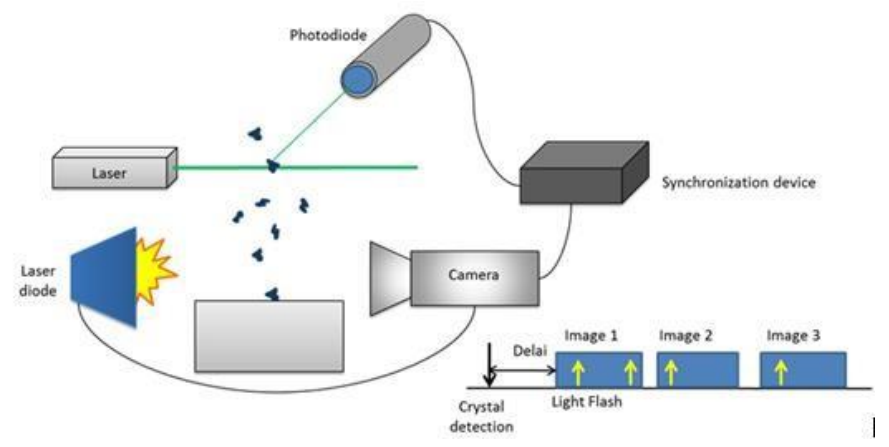

Figure 4. Particle detection system for splash visualization

For each impingement event, three successive images were recorded. Image 1 (see Figure 5) is designated to obtain the characteristics of the incident drop. For this purpose, during time that the camera shutter gate remains open the laser illuminates the drop twice. Through image processing, the size and velocity of drops can be measured before impact. The origin of the time $t_{0}=0 \mathrm{~s}$ corresponds to the beginning of the impact as far as could be determined by image processing. The other two images (Image 2 and Image 3 ) are matched with respect to the moment of impact $t_{0}$ and are used to document droplet impingement and splashing on the target surface. The image processing was performed with applications developed within ImageJ and Python.

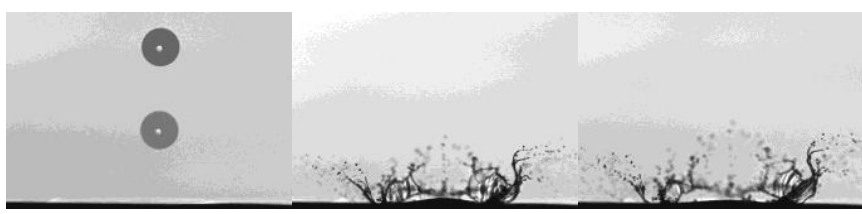

Image 1

Image 2

Image 3

Figure 5. Example of image sequence for a drop normal impact on a polished surface $(\mathrm{D}=260 \mu \mathrm{m} ; \mathrm{Vd}=40 \mathrm{~m} / \mathrm{s})$

During the splash visualization, several parametric studies were conducted with a clean aluminum surface (two roughnesses) and a surface covered with blotter paper. The parametric studies included variations in the air velocity for fixed sizes of drops. The objective was to explore the effect of drop size and velocity on the splashing behavior.

Page 4 of 11 


\section{Instrumentation for deposition rate}

Usually, in the literature [5], the quantity that is measured in the experiment is the collection efficiency $\beta_{\text {dep }}$ and it is measured using the blotter paper strips installed on the airfoils. Given that the impingement efficiency cannot be measured, it is not possible to extract the values of the deposition rate from the experimental data only. A numerical approach must be used additionally to determine the impingement efficiency, as done by Trontin and Villedieu [2].

In this paper the deposition rate, i.e., the fraction of liquid that remains on the wall after the impact, will be determined by direct measurement. The solution proposed here consists in the recovery of the liquid that settles on a small size surface and its weighing. The assembly consists mainly of a flat plate on which the drops are impinging.

The assembly is shown in the left picture of Figure 6. It consists of a removable target plate on which the drops are impinging. It has a planar rectangular shape with dimensions greater than the output section of the convergent, to receive as many incident drops as possible. The plate was mounted on the top of a liquid collector tank that can be tilted to study the influence of the impact angle on the deposition rate. The fraction of the drops deposited on the plate flows towards the edges and is recovered in the collector through two lateral drain slots. The secondary drops are assumed to be carried away by the air flow. The water was intended to flow into the drain slots but, in practice, it can bead at the edge of the slot and blow off the target assembly. Therefore, the edges of the collector were placed slightly above the target surface in order to capture as much deposited water as possible.

This plate is exposed to droplet impacts for a well measured period of time and the deposited mass flow rate $\dot{m}_{d e p}$ is compared to the impinging mass flow rate $\dot{m}_{i m p}$ to determine the average deposition rate:

$$
\varepsilon=\frac{\dot{m}_{d e p}}{\dot{m}_{i m p}}=\frac{\beta_{d e p}}{\beta_{i m p}}
$$

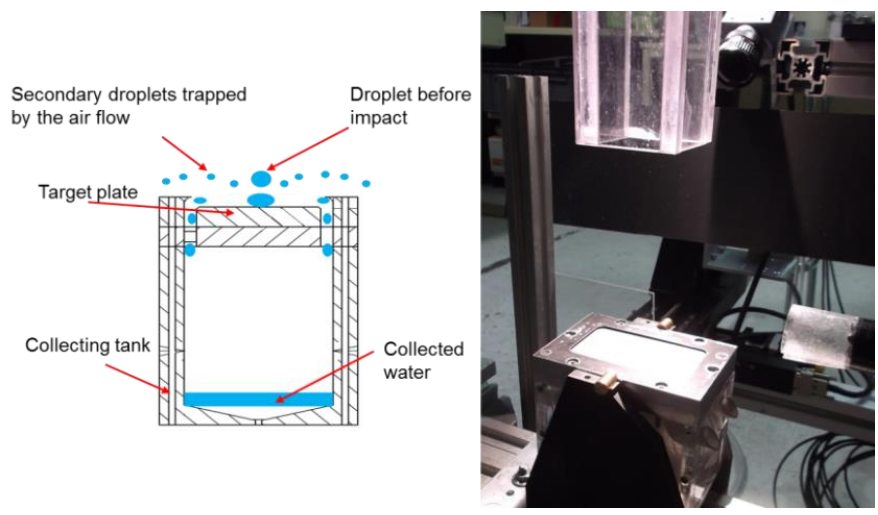

Figure 6. Instrumentation for deposition rate measurement

The implementation of this method was difficult because the measurements are very sensitive to external conditions. The first time it was necessary to evaluate precisely the impinging mass flow rate $\dot{m}_{i m p}$ that actually impacts the plate. The initial attempt to consider $\dot{m}_{i m p}$ equal to the injected mass flow of water led to erroneous results. Despite the careful design of the accelerating channel, it was Page 5 of 11 observed that the carrier airflow may disperse the drops and some of them impact the walls of the channel. Another source of water mass loss was the vaporization of drops from the injection to the impingement on the plate. The use of humidified air (relative humidity around $70 \%$ to avoid uncontrolled condensation) limited the latter effect. Finally, to correctly estimate the impinging mass flow rate, the same recovery/weighing method was used. Nevertheless, in this case the target plate was replaced by a honeycomb that sends the liquid flows directly towards the collecting tank.

The first tests demonstrated the need to apply a very rigorous data acquisition method. Hence, to verify the stability of the operating conditions, at the beginning and end of the test sequence the impinging mass flow rate was measured as the mean value over three successive runs. The deposited mass flow rate was equally measured as the mean value over at least three runs. Non-physical or aberrant values were removed from the statistical analysis. During the acquisition sequence, the air mass flow rate, temperature and humidity were recorded continuously and images of the drops before impact were acquired to monitor their size and velocity values.

For tests on a blotter paper substrate, the accumulation time interval was very short $(<5 \mathrm{~s})$ to avoid the saturation of the blotter paper.

\section{Results and discussions}

The airflow velocity at the exit of the square cross section tube was measured using a Pitot probe in the absence of liquid injection. Measurements were performed for mass flow rates ranging from 20 to $250 \mathrm{~g} / \mathrm{s}$ (Figure 7) on the axis of the channel, i.e., where the liquid phase is present. The droplet sizes and velocities before impact were measured by processing the images recorded with the JAI camera. For all air velocities different slip velocities were measured between the air and the drops before the impact.

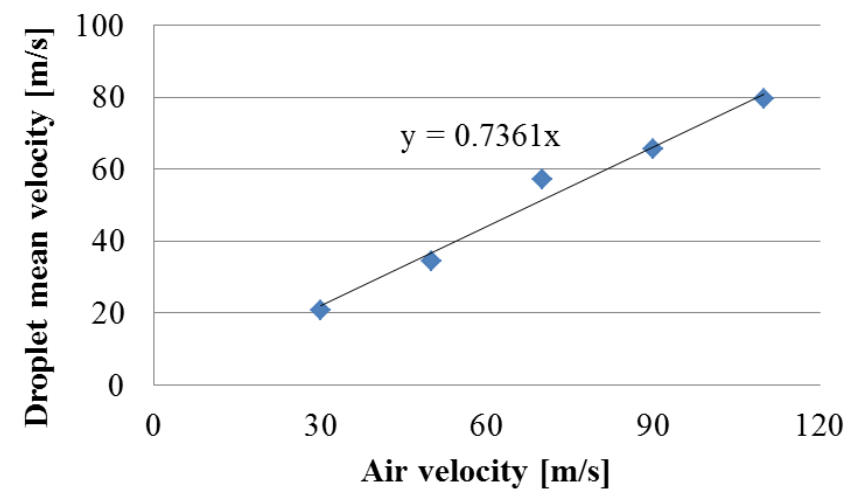

Figure 7. Droplet velocity as a function of the air speed

The top graph on Figure 8 shows the 11-bin droplet size distributions for each set. The mean diameter of the drops has a relatively reduced variation with the air velocities. The bottom graph of the same figure shows the impact velocity distributions measured from the imaging data as a function of the air velocity in the end section of the tunnel. 

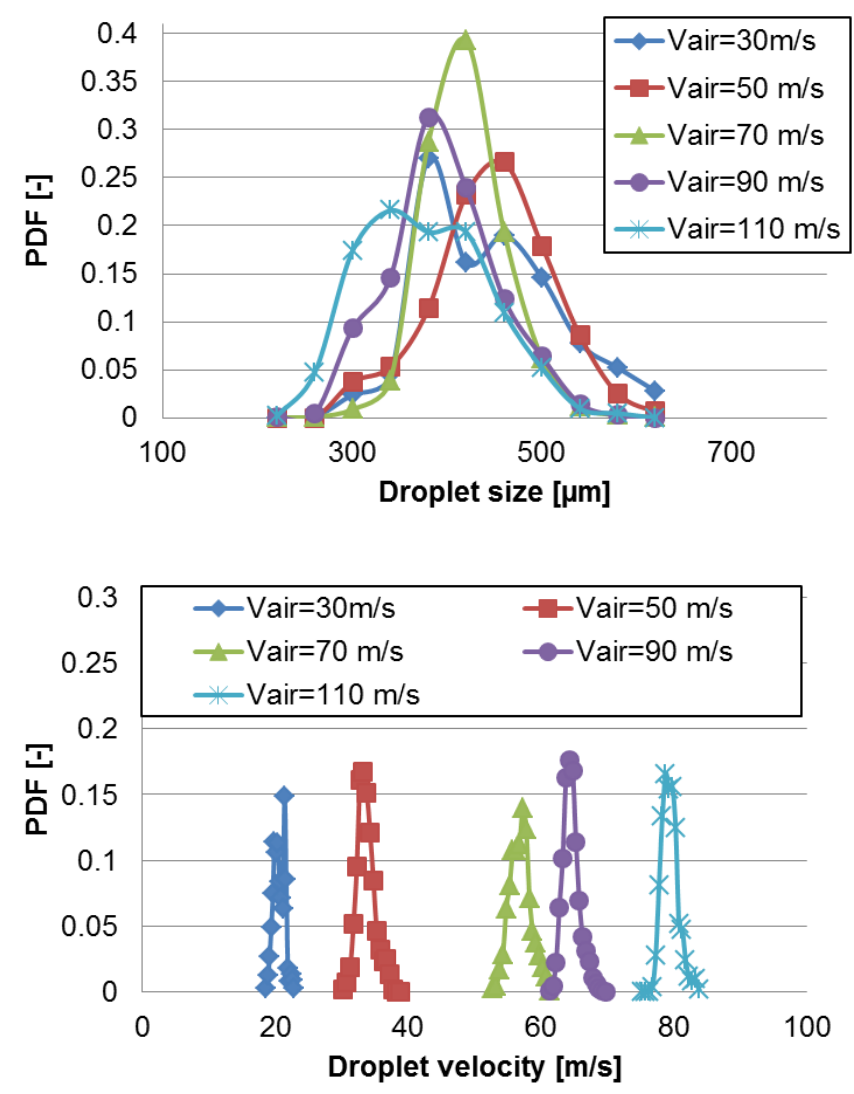

Figure 8. Droplet sizes and velocities before impact.

It must be added that the drops do not all have a spherical shape. Although for small drops travelling at small velocities this is always valid, for large drops traveling at high velocities a small deformation of their shape was observed.

\section{Results for droplet splashing}

The results presented in this section are qualitative and are based on the analysis of images recorded with the JAI camera. The images presented are on a plane normal to the impingement surface and to its turning axis. The flow in all cases is from top to bottom that corresponds to a normal impact. The contours that appear blurred in the figures correspond to liquid structures that are out of the depth of field.

A high number of images of impact events have been recorded. Of these, a selection of about one hundred was analyzed for each class of sizes. In the following figures only four of them are presented for each operating condition. It must be noted that each image corresponds to a different impact event. Each line of pictures shows the droplet at different moments during the impact. The time elapsed between images has been adapted as a function of the drop velocity, in order to follow the impact phenomena.

Backlit images were recorded for driving air speeds ranging from 30 $\mathrm{m} / \mathrm{s}$ to $110 \mathrm{~m} / \mathrm{s}$, and for an impact angle of $90^{\circ}$. The images presented in the following figures correspond to drops with a diameter of $375 \pm 15 \mu \mathrm{m}$.

Page 6 of 11
Figure 9 shows instantaneous images of drops splashing on the polished surface. Each row corresponds to a different impact velocity that increased from $25 \mathrm{~m} / \mathrm{s}$ to $80 \mathrm{~m} / \mathrm{s}$ for a fixed size of drops.

It must be noted that, for a clean surface, all of the recorded impact events correspond to drop impacts on a dry surface. The combined effect of the small size of the target plate, the law droplet density number and the presence of the airflow prevents the formation of a liquid film on the impinging surface. It was observed that between two different impact events the entire mass of water was completely blown out. A different behavior may be observed for the blotter paper covered surface. For long spraying times (> $5 \mathrm{~s}$ ) water accumulates on the substrate and the impact dynamics corresponds to the impact on a liquid film. To compare the visualizations for the different substrates the spraying time was limited to $5 \mathrm{~s}$ for the blotter paper covered surface.

The main observation from all of the recorded images is that the droplet splashing is evident for all impinging surfaces even though it is different for an absorbent substrate.

Drops seem to splash via two different mechanisms: corona splash and prompt splash. For low impinging velocities the impact dynamics are very similar to those described by Mundo et al. [12]. In the first images, with a relatively low impact velocity, the corolla formation phenomenon is visible and atomization results from the fragmentation of the liquid film forming the corolla. Hence, as the droplet touches the surface, a liquid film spreads outwards. A corona around the deformed droplet is formed and grows in time as the droplet fluid continues to feed the film. Once the droplet has completely disappeared (there is no more volume flow rate into the wall film), the corona, having been stretched in its radial expansion, now has less fluid feeding the film and becomes thinner. An instability develops and leads to a circumferential wreath, which propagates upward in the corona and finally results in a disintegration into secondary drops.

When the speed of impact increases, this phenomenon disappears, the atomization occurs earlier and closer to the wall, and the created drops become smaller. For high impingement speeds, we see a (prominent) prompt splash with many drops ejected at large angles from the spreading contact line. On increasing the impact velocities the ejected cloud of drops becomes denser and the size of the secondary drops seems to decrease significantly.

For higher impingement velocities, a rigorous disintegration of the liquid film is observed. In this case, the corona and the associated instabilities are no longer identifiable. This behavior is similar to that observed for drops impinging on a rough aluminum surface (Figure 10). Furthermore, the concentration of the secondary drops is much higher for great velocities and rough surfaces.

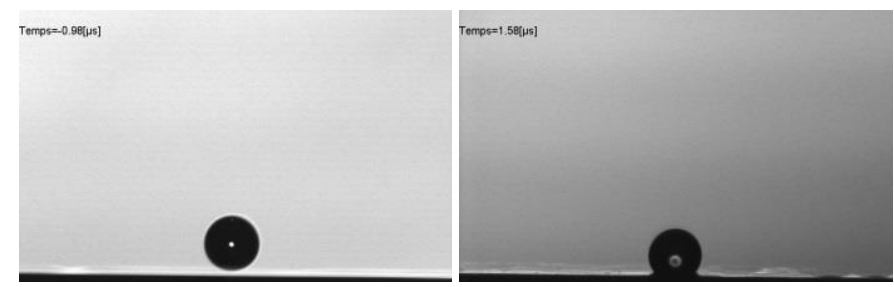



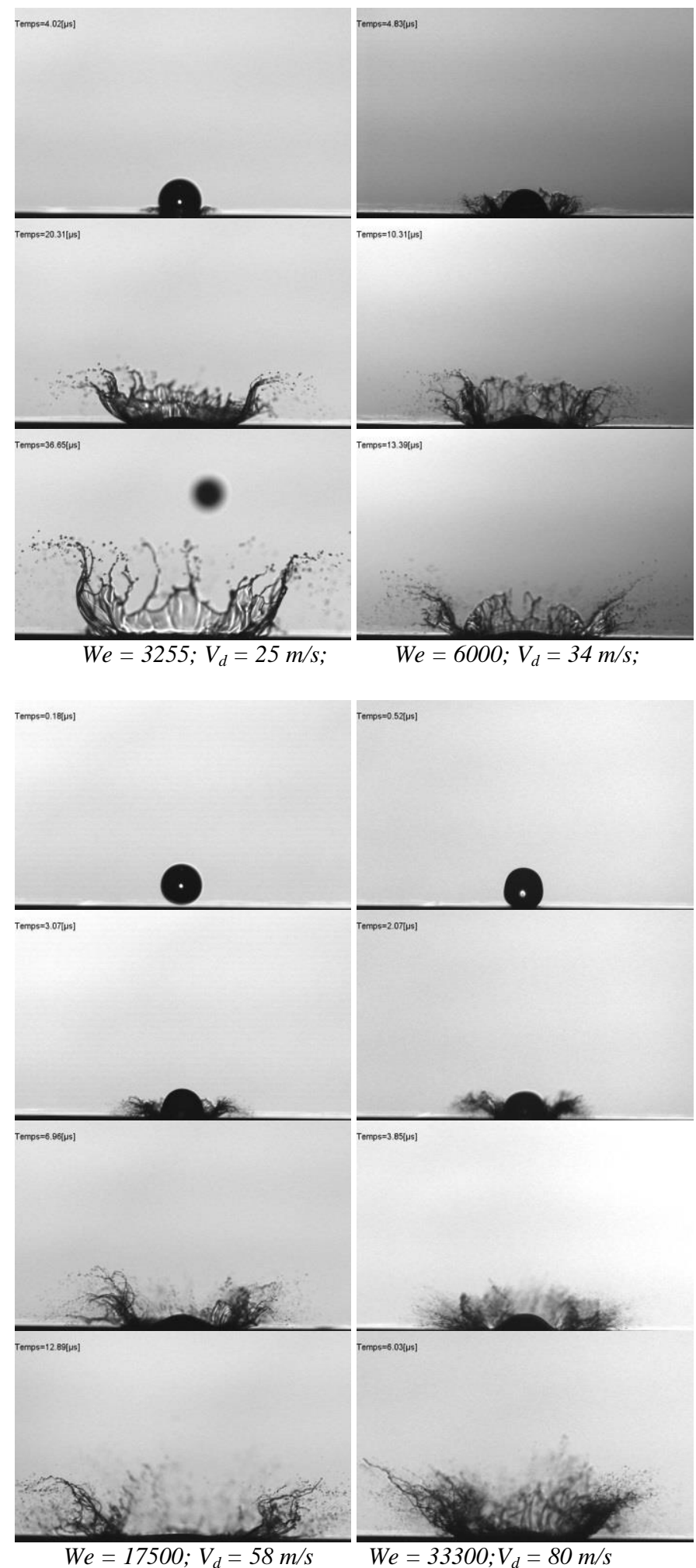

Figure 9. Droplet normal impact for different velocities on a polished aluminum surface (A series of image sequences given by order of droplet velocity); $\mathrm{D}=375 \mu \mathrm{m}$

Figure 13 shows an identical drop under the same conditions impacting a rough aluminum surface. The roughness doesn't seem to change the drop dynamics during the impact. The images are very similar to those corresponding to the impact on the polished surface but for a high velocity.

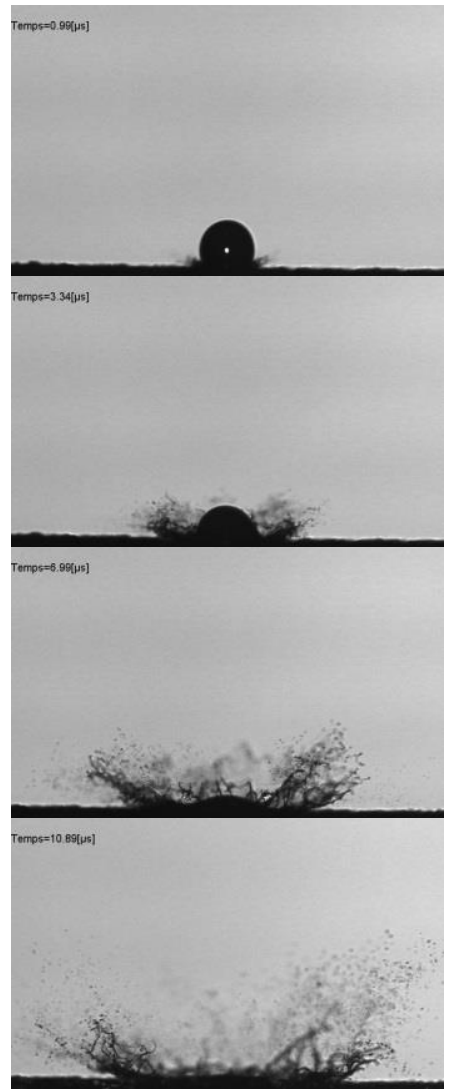

$W e=17500 ; V_{d}=58 \mathrm{~m} / \mathrm{s}$

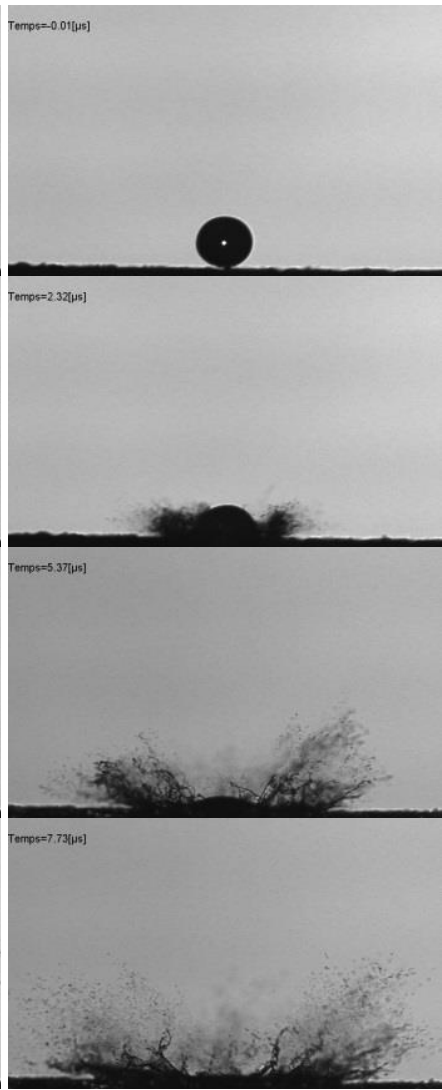

$W e=22000 ; V_{d}=65 \mathrm{~m} / \mathrm{s}$
Figure 10. Droplet normal impact for different velocities on a rough aluminum surface (a series of image sequences given by order of droplet velocity); $\mathrm{D}=$ $375 \mu \mathrm{m}$

The dynamics of impact on the blotter paper are summarized in Figure 11. Surface absorption significantly affects the splashing. For the aluminum surfaces a significant volume of water appears to be ejected from the impact site. Nevertheless, for a surface covered with blotter paper it seems that the ejected volume of water is negligible. The blotter paper acts as an absorbent and captures almost all of the incident volume of water.

Examination of a great number of images obtained with and without blotter paper show different results, which is different from the conclusions of Papadakis et al. [6]. Contrary to this reference, for the blotter paper test the drop splashing was not eliminated but it is very different in terms of secondary drop generation. These differences between drop splashing on a clean surface versus drop splashing on blotter paper may significantly impact the development of models for icing applications, in so far as, to date, the two impingement scenarios are considered almost identical. This conclusion will be strengthened by quantitative measurement of deposition rates on the two surfaces. 

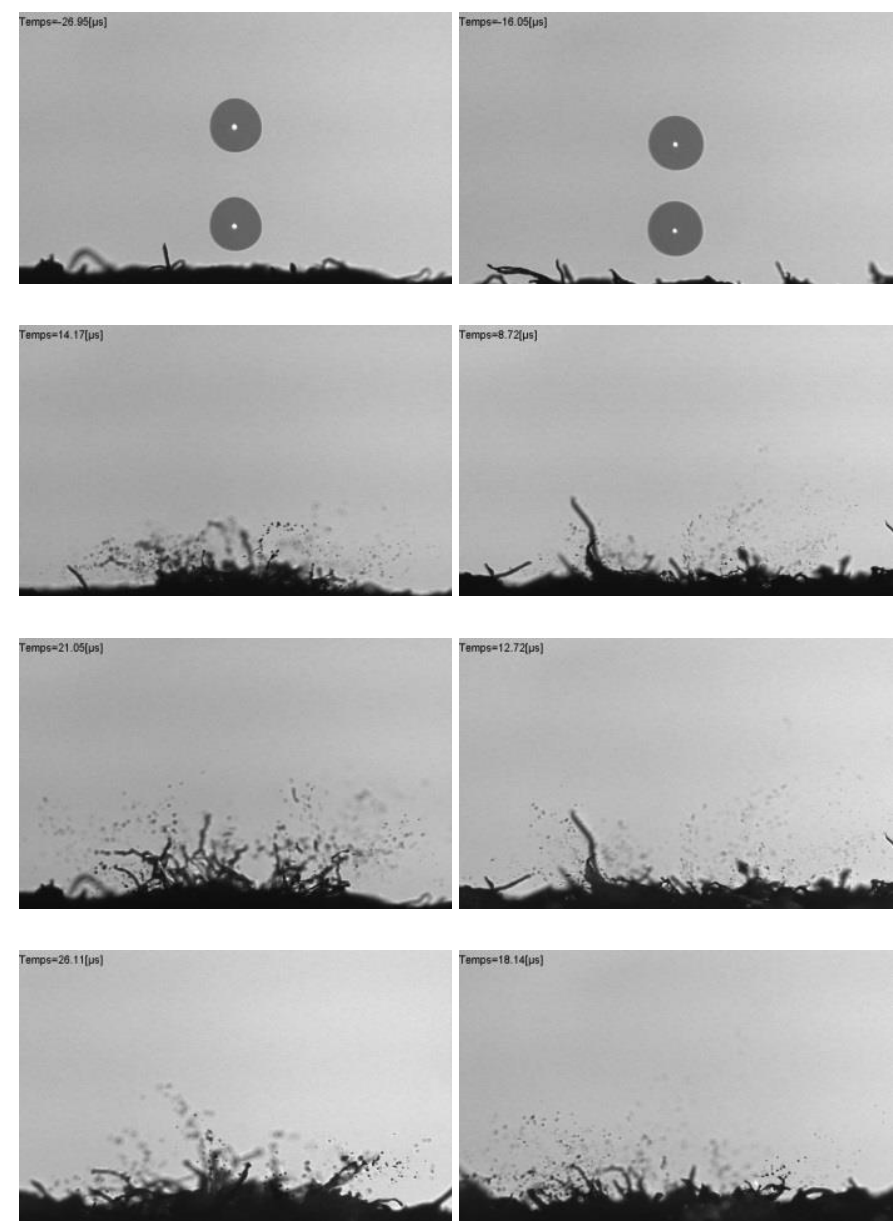

$W e=6000 ; V_{d}=34 \mathrm{~m} / \mathrm{s} W e=17500 ; V_{d}=58 \mathrm{~m} / \mathrm{s}$

Figure 11. Droplet normal impact for different velocities on a blotter paper covered surface (A series of image sequences given by order of droplet velocity); $\mathrm{D}=375 \mu \mathrm{m}$

\section{Results on deposition rate}

The second aspect evaluated within this work is the deposition rate $\varepsilon$ for different drop velocities, impingement surfaces, and incidence angles. The angles of incidence and impact are defined as in Figure 12.

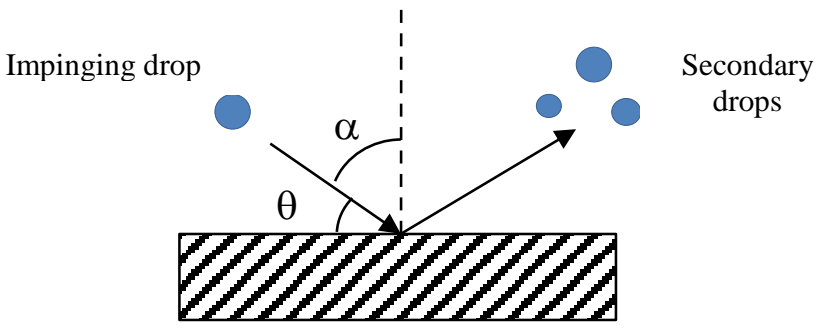

Figure 12. Definition of the angle of incidence $\theta$ and the impact angle $\alpha$

Table 1 lists the operating conditions when a clean aluminum impingement surface is used. For these measurements 19 operating conditions were tested. Of the order of three to six tests were performed for identical conditions and the median values were used in the results. For each run the value does not deviate by more than $10 \%$ from this median value. A total number of 72 runs were acquired to determine the deposition rate for the clean aluminum surface.

\begin{tabular}{|c|c|c|c|c|c|}
\hline$\theta\left[^{\circ}\right]$ & $\mathbf{V}_{\mathbf{d}}[\mathbf{m} / \mathbf{s}]$ & $\mathbf{W e}_{\mathbf{n}}[-]$ & K [-] & Ca $[-]$ & $\begin{array}{c}\text { Number of } \\
\text { tests }\end{array}$ \\
\hline \multirow{5}{*}{90} & 27.2 & 3853 & 3970 & 0.37 & 3 \\
\hline & 40 & 8333 & 6462 & 0.55 & 3 \\
\hline & 54 & 15188 & 9360 & 0.74 & 9 \\
\hline & 70.5 & 25887 & 13062 & 0.97 & 6 \\
\hline & 81 & 34172 & 15537 & 1.11 & 3 \\
\hline \multirow{5}{*}{70} & 27.2 & 3403 & 3674 & 0.37 & 3 \\
\hline & 40 & 7359 & 5950 & 0.55 & 3 \\
\hline & 54 & 13411 & 5560 & 0.74 & 6 \\
\hline & 70.5 & 22860 & 12850 & 0.97 & 3 \\
\hline & 81 & 30175 & 14375 & 1.11 & 3 \\
\hline \multirow{5}{*}{50} & 27.2 & 2261 & 2845 & 0.37 & 3 \\
\hline & 40 & 4890 & 4609 & 0.55 & 3 \\
\hline & 54 & 8912 & 6708 & 0.74 & 6 \\
\hline & 70.5 & 15190 & 9360 & 0.97 & 3 \\
\hline & 81 & 20053 & 11135 & 1.11 & 3 \\
\hline \multirow{4}{*}{20} & 27.2 & 451 & 1040 & 0.37 & 3 \\
\hline & 40 & 975 & 1682 & 0.55 & 3 \\
\hline & 70.5 & 3028 & 3416 & 0.97 & 3 \\
\hline & 81 & 3997 & 4046 & 1.11 & 3 \\
\hline
\end{tabular}

Table 1. Test matrix for a clean aluminum impingement surface

Table 2 shows the operating conditions when the impingement on a blotter paper substrate was investigated. As for the clean aluminum surface, three to five tests were run for each operating point. For the 17 conditions listed in the table a number of 60 measures were performed. Overall, considering the runs for the determination of the impingement mass flow rate a total number of 160 tests were run for the deposition rate investigation.

\begin{tabular}{|c|c|c|c|c|c|}
\hline$\theta\left[{ }^{\circ}\right]$ & $\mathbf{V}_{\mathbf{d}}[\mathbf{m} / \mathbf{s}]$ & $\mathbf{W e}_{\mathbf{n}}[-]$ & $\mathbf{K}[-]$ & $\mathbf{C a}[-]$ & $\begin{array}{c}\text { Number of } \\
\text { tests }\end{array}$ \\
\hline \multirow{4}{*}{90} & 27.2 & 3853 & 3970 & 0.37 & 3 \\
\cline { 2 - 6 } & 40 & 8333 & 6462 & 0.55 & 3 \\
\cline { 2 - 6 } & 54 & 15188 & 9360 & 0.74 & 4 \\
\cline { 2 - 6 } & 70.5 & 25887 & 13062 & 0.97 & 5 \\
\cline { 2 - 6 } & 81 & 34172 & 15537 & 1.11 & 3 \\
\hline \multirow{4}{*}{70} & 27.2 & 3403 & 3674 & 0.37 & 3 \\
\cline { 2 - 6 } & 40 & 7359 & 5950 & 0.55 & 3 \\
\cline { 2 - 6 } & 70.5 & 22859 & 12850 & 0.97 & 2 \\
\cline { 2 - 6 } & 81 & 30175 & 14375 & 1.11 & 3 \\
\hline \multirow{4}{*}{50} & 27.2 & 2261 & 2845 & 0.37 & 3 \\
\cline { 2 - 6 } & 54 & 8912 & 6708 & 0.74 & 5 \\
\cline { 2 - 6 } & 81 & 20053 & 9360 & 0.97 & 3 \\
\hline \multirow{4}{*}{20} & 70.5 & 15191 & 11135 & 1.11 & 3 \\
\cline { 2 - 6 } & 27.2 & 451 & 1040 & 0.37 & 3 \\
\cline { 2 - 6 } & 40 & 4890 & 1682 & 0.55 & 8 \\
\cline { 2 - 6 } & 70.5 & 3028 & 3416 & 0.97 & 3 \\
\hline
\end{tabular}

Table 2. Test matrix for a blotter paper impingement surface

For the blotter paper tests a principal concern was the saturation with liquid. It was observed that, beyond a critical value, the accumulated mass of water becomes non-linear with the accumulation time. In

Page 8 of 11 
addition, the accumulation of an important quantity of liquid severely influences the mechanical properties of the blotter paper layer with consequences on the measurement precision and reproducibility, especially when high impact velocities are investigated.

The evolution of the deposition rate versus the accumulation time for a normal impingement was recorded for different operating conditions. It was observed that for an accumulation time of less than $10 \mathrm{~s}$, the deposition rate is equal to unity within an uncertainty band of $10 \%$. For longer accumulation times the deposition rate decreases severely up to $40 \%$. Following this analysis it was decided to use for all the test runs an accumulation time of $5 \mathrm{~s}$.

Figure 13 shows the evolution of the deposition rate with the normal Weber number $W e_{n}$ for different incidence angles. The two graphs correspond to the clean polished aluminum (top picture) and blotter paper substrate (bottom picture), respectively.

The tests performed on a clean aluminum surface show that, for small values of the $W e_{n}$ (usually less that 6000), the deposition rate is an increasing function of $W e_{n}$. For $6000<W e_{n}<20000$ a variation of $\varepsilon$ only with the incident angle is observed while for $W e_{n}>20000$ the values of $\varepsilon$ are very close $(\varepsilon \sim 0.6)$ for all of the incident angles.

When the blotter paper is used, the measured deposition rate is equal to unity for incidence angles greater than $50^{\circ}$. This result suggests that all of the impinging drops deposit onto the surface, which is consistent with the observations carried out by backlit imagery for this substrate. Due to the absorption proprieties of the blotter paper, the water drops are absorbed and the splashing does not occur. It must be noted that the values $\varepsilon>1$ on the right side picture have no physical meaning. They are the result of the measurement uncertainties, which are smaller than $10 \%$ in RMS values. For small incidence angles $\left(\theta=20^{\circ}\right)$ a decrease of $\varepsilon$ with the normal incident velocity (expressed through the normal Weber number) is observed. These particular results can be explained by the fact that, for highly oblique impingements, the impact regime does not correspond to a splash, but rather to a rebound, which limits the amount of water that is deposited on the wall. Given that the same behavior was not observed for the clean aluminum surface, it is believed that the surface quality of the blotter paper may enhance the rebound mechanism.

From a physical point a view, the existence of two different impact regimes was explained by Trontin and Villedieu [2]. For small angles of incidence the capillary forces, which tend to promote drops sticking to the wall, are balanced by the tangential inertial forces, which tend to tear the droplet off the wall. This is in agreement with the observations carried out in the first part of the document. When a droplet impinges the wall with a law impact angle it tears on the surface and a large amount of liquid is carried away by the flow so the deposition is low.
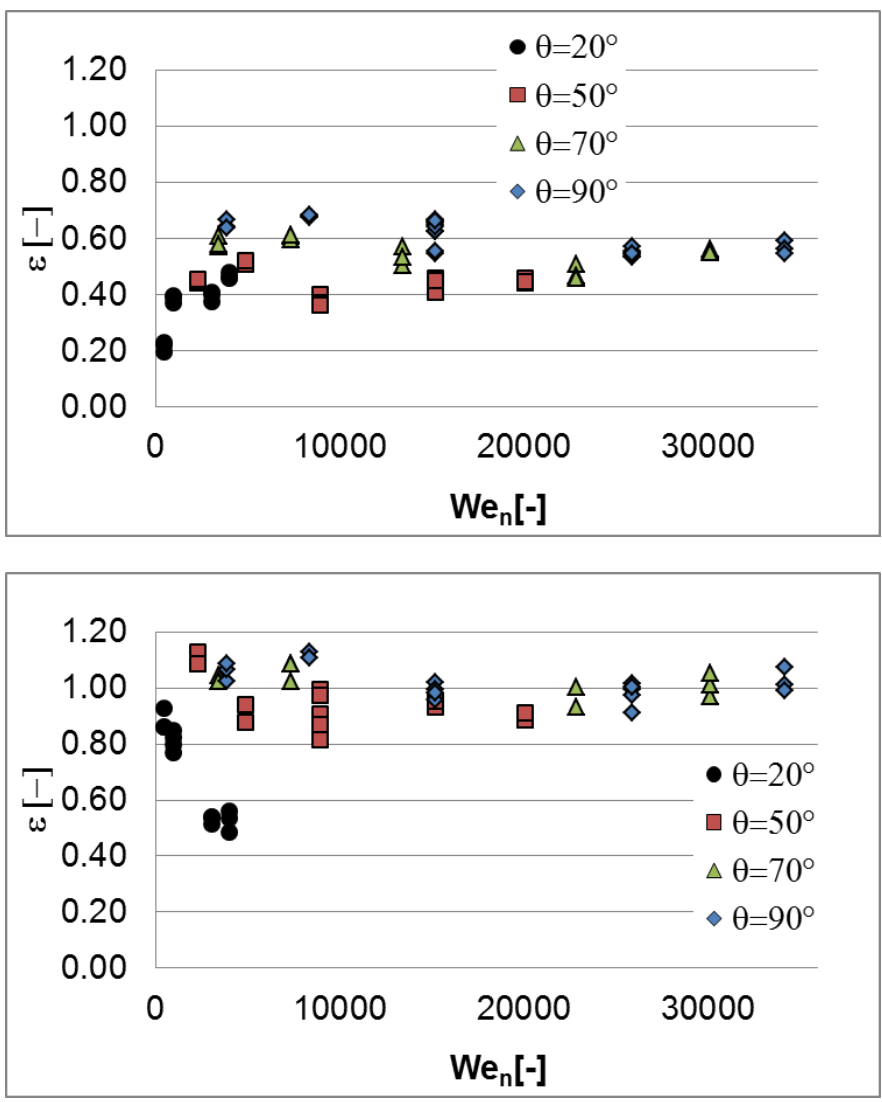

Figure 13. Deposition rate measurement results as a function of the normal Weber number for clean aluminum (left side picture) and blotter paper (right side picture).

The measured values of the deposition rate are compared in Figure 20 with the values obtained by Papadakis et al. [5], [6] for different airfoil profiles. Nevertheless, the quantity experimentally measured by Papadakis et al. is the collection efficiency $\beta_{d e p}$. Given that the impingement efficiency $\beta_{\text {imp }}$ is not experimentally available, it is not possible to compute the values of the deposition rate from the experimental data only. Within this work the values of $\beta_{i m p}$ are those calculated by Trontin and Villedieu [2] for different test conditions and impingement geometries.

The measured values of the deposition rate $(\varepsilon)$ are plotted in Figure 14 versus the impingement angle. Three series of data are compared:

1. The reference data of Papadakis et al. obtained with the blotter paper for aerodynamic profiles (small square symbols). From the available data base only the tests performed for large drops (MDV=137, 154 and168 $\mu \mathrm{m}$ ) were used for the comparison.

2. The ONERA data obtained for a plate covered by blotter paper (red circles)

3. The ONERA data obtained for a clean and polished aluminum plate (blue triangles)

Despite a significant amount of scattering of the reference data, the existence of two impinging regimes is obvious. The first regime, obtained for low values of $\theta$, corresponds to a linear evolution of $\varepsilon$ with $\theta$. For the second regime $\varepsilon$ is independent of $\theta$ ( $\varepsilon$ between 0.8 and 1). The critical angle between the two regimes oscillates between $18^{\circ}$ and $25^{\circ}$. 
The experimental method proposed within this document is assessed comparing the deposition rates obtained on the blotter paper substrate with the reference data. For $\theta>50^{\circ}$ a total deposition is obtained $(\varepsilon \sim 1)$ for the incident water drops, result that is slightly greater than that of Papadakis et al. For $\theta=20^{\circ}$, the scattering in the ONERA data (evolution of $\varepsilon$ with the impacting normal velocity) renders the comparison difficult. Nevertheless, the values reported here are within the cloud of data reported by Papadakis et al. for the same angle of incidence.

The comparison of $\varepsilon$ measured within this work for a clean aluminum plate with the Papadakis et al. reference values shows important differences both in the critical impact angle $\left(\theta \sim 45^{\circ}\right.$ in our tests) and in the maximal value of the deposition rate ( $\varepsilon$ between 0.6 and 0.8 ).

In our opinion, this is not an unexpected result because, as it was shown by visualization, the splashing behavior is greatly dependent on the nature of the impingement surface: clean aluminum or blotter paper. From the results, it is strongly believed that the measurement of the deposition rate performed with the blotter paper is not representative of a clean aluminum surface. Nevertheless, the discussion is ongoing for the impact on an iced surface.

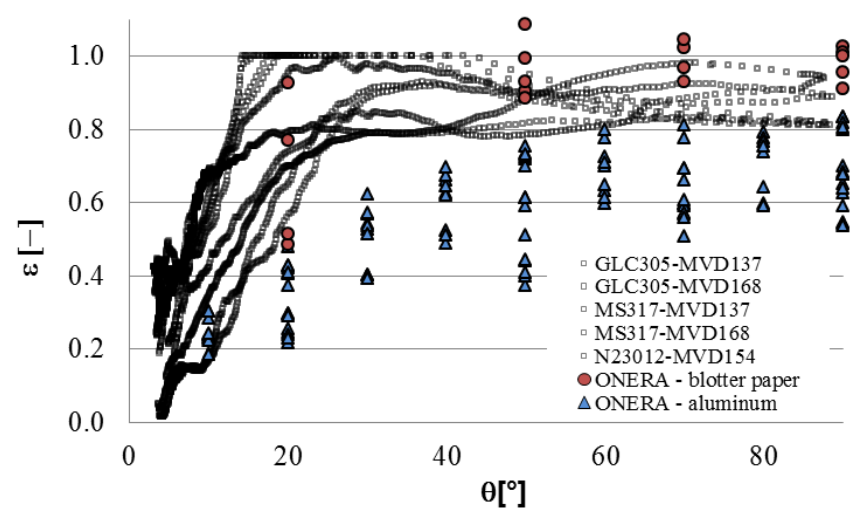

Figure 14. Deposition rate versus the impact angle. Three series of data are compared: Papadakis et al. [5][6] (square symbols), ONERA for an aluminum plate (blue triangles), ONERA for blotter paper (red circles).

\section{Conclusions}

The objective of the study was to improve the understanding of large drops splashing for the development of more accurate physical models for the ice accretion due to SLD. For that an ONERA new test facility was used to analyze the impact behavior of high-speed drops.

Within this work backlighted visualization and recovery/weighing techniques have been applied for documenting droplet impingement, splashing and deposition rate on different surfaces.

The droplet impingement and splashing was visualized using a video camera. The images were used to determine the drops size and velocity before impact. The image analysis revealed that for a clean aluminum surface the splashing creates some characteristic features as "coronas" and "prompts" generating large number of smaller drops which are rejected into the air stream. For the absorbent surface the splashing is modified. When the drop impacts on a blotter paper surface neither splash corona nor prompt is formed and a negligible amount of secondary drops is produced.

Page 10 of 11
For the measurement of the deposition rate a recovery/weighing method was used. Contrary to the results of Papadakis et al. [5], [6] it was established that the dynamics of drop impingement on blotter paper is not the same as impingement on a clean surface.

\section{References}

1. Wright, W. B., "Validation results for LEWICE 3.0", 43 ${ }^{\text {rd }}$ AIAA Aerospace Sciences Meeting and Exhibit, AIAA paper 2005-1243, 2005

2. Trontin, P., Villedieu, P., "Revisited model for supercooled large droplet impact onto a solid surface", Journal of Aircraft, 2016, doi: 10.2514/1.c0340952

3. Sabri, F., Trifu, O., Paraschivoiu, I., "In-flight ice accretion simulation in SLD conditions", $25^{\text {th }}$ AIAA Applied Aerodynamics Conference, Miami, Florida, 2007

4. Li, H., Roisman, I., Tropea, C., "Physics of supercooled large drops (SLD): literature review, extreme icing environment", EXTICE project, WP2 intermediate report, Darmstadt, 2009

5. Papadakis, M., Hung, K. E., Vu, G. T., Yeong, H. W., Bidwell, C. S., Breer, M. D., Bencic, T. J., "Experimental investigation of water droplet impingement on airfoils, finite wings, and an sduct engine inlet", NASA Tr 2002-211700, 2002

6. Papadakis, M., A. Rachman, S. C. Wong, Hung, K. E., Vu, G. T., Bidwell, C. S., "Experimental study of supercooled large droplet impingement effects", DOT/FAA/AR - 03/59 Report, Office Of Aviation Research Washington, D.C., 2003

7. Tan, S., C., Papadakis, M., Miller, D., Bencic, T., Tate, P., Laun, M., C., "Experimental study of large drops splashing and breakup", $45^{\text {th }}$ AIAA Aerospace Sciences Meeting And Exhibit, Reno, Nevada, 2007

8. Tan, S. C., "A tentative mass loss model for simulating water droplet splash", AIAA Conference AIAA-2004-410, Reno, 2004

9. Yarin, A., L., "Drop impact dynamics: splashing, spreading, receding, bouncing", Annual review of fluid mechanics, no 38, pp 159-192, doi: 10.1146/annuarev.fluid.38.050304.092144

10. Josserand, C., Thoroddsen, S., "Drop impact on a solid surface", Annual review of fluid mechanics, no 48, pp 365-391, 2016, doi: 10.1146/annuarev-fluid-122414-034401

11. Rein, M., "Phenomena of liquid drop impact on solid and liquid surfaces", Fluid Dynamics Research, vol. 12, pp 61-93, 1993

12. Mundo, C., Sommerfeld, M., Tropea, C., "Droplet-wall collisions: Experimental studies of the deformation and breakup process", Int. J. Multiphase Flow, vol. 21, no. 2, pp. 151-173, 1995

13. Guo, Y., Lian, Y., Sussman, M., "Investigation of drop impact on dry and wet surfaces with consideration of surrounding air", Physics of Fluids, 28, 073303, 2016

14. Cossali, G. E., Coghe, A., Marengo, M., "The impact of a single drop on a wetted solid surface", Experiments in Fluids, vol. 22, no. 6, 1997, pp. 463-472, doi:10.1007/s003480050073

15. Rioboo, R., Tropea, C., Marengo, M., "Outcomes from a drop impact on solid surfaces", Atomization and Sprays, vol. 11, pp. 155-165, 2001, doi: 10.1615/atomiz.v11.i2.40

16. Xu, L., Barcos, L., Nagel, S., R., "Splashing of liquids: interplay of surface roughness with surrounding gas", Physical Review, E $76,066311,2007$

17. Latka, A., Strandburg-Peshkin, A., Driscoll, M., M., Stevens, C., S., Nagel, S., R., "Creation of prompt and thin-sheet splashing by varying surface roughness or increasing air pressure", Physical Review Letters, PRL 109, 054501, 2012

18. Berthoumieu, P., "Experimental study of supercooled large drops impact in an icing wind tunnel." AIAA 2012-3130, AIAA Conference, New Orleans, 2012 
19. Berthoumieu P., Déjean, B., "Experimental investigation of SLD impact phenomena", $7^{\text {th }}$ European Conference for Aeronautics and Space Sciences -EUCASS, Milan, Italy, 2017

\section{Contact Information}

BODOC Virginel : virginel.bodoc@ onera.fr.

\section{Acknowledgments}

This work was conducted in the frame of the French research program PHYSICE funded by the Direction Générale de l'Aviation
Civile (DGAC). Many thanks to Pierre Trontin for supporting the reference data base.

\section{Definitions/Abbreviations}

SLD

Supercooled large droplet 